$11-1-2014$

\title{
Revisiting the use of secondary task reaction time measures in telepresence research: exploring the role of immersion and attention
}

\author{
Cheryl C. Bracken \\ Cleveland State University, c.bracken@csuohio.edu \\ Gary Pettey \\ Cleveland State University, g.pettey@csuohio.edu \\ $\mathrm{Mu} \mathrm{Wu}$
}

Follow this and additional works at: https://engagedscholarship.csuohio.edu/clcom_facpub

Part of the Communication Commons

How does access to this work benefit you? Let us know!

Publisher's Statement

"The final publication is available at Springer via http://dx.doi.org/10.1007/s00146-013-0494-7

\section{Recommended Citation}

Bracken, C. C., Pettey, G., \& Wu, M. (2014). Revisiting the use of secondary task reaction time measures in telepresence research: exploring the role of immersion and attention. AI \& SOCIETY, 29(4), 533-538.

https://doi.org/10.1007/s00146-013-0494-7

This Article is brought to you for free and open access by the School of Communication at EngagedScholarship@CSU. It has been accepted for inclusion in Communication Faculty Publications by an authorized administrator of EngagedScholarship@CSU. For more information, please contact library.es@csuohio.edu. 


\title{
Revisiting the use of secondary task reaction time measures in telepresence research: exploring the role of immersion and attention
}

\author{
Cheryl Campanella Bracken Gary Pettey \\ Mu Wu
}

\begin{abstract}
In this experimental study, we use secondary task reaction time (STRT) to measure Attention to a media presentation and compare STRT to traditional self-report measures of Telepresence (immersion, social reality, spatial presence, and transportation) and enjoyment. Further, we compare the STRT measure with the composite items of Telepresence-Immersion. The results indicate that STRT may be useful for measuring some sub-dimensions of Telepresence. Implications are discussed.
\end{abstract}

\section{Introduction}

Telepresence has primarily been studied with self-report (pencil-and-paper) measures gathered after an exposure to a media experience. While numerous researchers have commented on the necessity of more objective measures over the past decade (Alcañiz et al. 2009; IJsselsteijn et al. 2000), few have proven fruitful. This study will incorporate the concept of Attention, measured by secondary task reaction time (STRT), in an attempt to clarify the self-report Telepresence measures.

\subsection{Telepresence}

There is general agreement that Telepresence is "the perceptual illusion of non-mediation" (Lombard and Ditton 1997. Para 2). Bracken and Pettey more recently developed the following definition based on their work with popular media and Telepresence: Telepresence is a "psychological state in which media users voluntarily suspend the experience of mediation in order to feel a sense of connection with the mediated content they are using (i.e.., connection to characters, involvement in the story line)" (Bracken et al. 2010. pg. 2; Pettey et al. 2010).

1.2 Telepresence and the nature of reality, concepts, and the virtual

Pinker (2007) argues that all communication is the ability to paint a picture in another's mind. Biocca (2003) refers to evolutionary primacy playing a role in shifts in Telepresence. He suggests that the "ability to shift ones' spatial presence must be based on mechanisms that most likely served an evolutionary value" (pg. 4). Biocca argues that we can conceive of situations when our ancestors needed to conceptualize/visualize or "experience the presence" of a place to explain a past or plan a future action. Drawing a map in the dust or using a rock as symbol for a mountain has evolutionary value when planning a hunt or giving directions. Biocca suggests this ability might be linked to the beginning of self-consciousness as well as embedding that self-consciousness within a social context.

Telepresence is based on our ability to conceptualize/ visualize these types of images in our mind. There is an interaction between the real world imagery, the virtual imagery, and our mental imagery. Biocea suggests there are three poles: physical space, mental imagery space, and 
ultimately (and humanly), virtual space. For example, a hunter experiences a meadow with game. The hunter visualizes, models, and remembers key features (i.e., conceptualizes the space), and then he shares the conceptualized space with others using abstractions (e.g., drawings). This virtual space can now be shared by individuals who have not physically experienced the space. Plans can be made for the real physical space by using the virtual space and sharing the original hunter's concepts (i.e., mentalization models). By acknowledging that individuals have the capability to create images in their own minds of some actual (or even conceived of) physical space, and communicate these images to others through abstractions (words or other symbols), it explains the ease with which our minds accept the technological creation of environments (space) and experiences. Further, that we do this with actual environments and experiences allow us to move into "created" ones and experience them as real. Our ability to represent imagery has obviously surpassed drawing in the dirt and we can now "share" the images we create using cameras, computers, and software with millions.

Such a three-pole model asserts a case, as well, of potentially divided Attention. When energy is divided across the poles (as it might be during initial learning), overall reported levels of Telepresence is low. Consider the transference of knowledge about how to walk through an actual physical space full of pylons, which must be reconfigured mentally when learning how to drive a vehicle through them. Learning to control a boat through water or a plane through air must involve some unlearning driving and turning a car. During that learning period, Telepresence would be low as attention is scattered across poles. One would see the a similar learning curve learning to use a video controller through a video game as the individual must merge physical experiences with conceptualized locomotion (controller) with the virtual space of the video game. Only once one has mastered the controller can Telepresence ensue (with skill being a necessary rather than a sufficient causal condition).

Conversely, as spatial attention increases (attention at a given pole), an individual may not have the resources to maintain different spatial models (at other poles). Under conditions where action in space involves high spatial attention to sensory stimuli (e.g., learning a new motor task or during a fight-or-flight), spatial presence is focused and undivided. When an individual's skills are being challenged whether in real space (e.g., driving in traffic during a snowstorm), in cognitive space (e.g., concentrating on detailed reading material) or in virtual space (e.g., being shot at in a combat centered video game), the level of mental energy required for processing of sensory cues approaches maximum. Any attempt to process away from that "pole" could result in failure as processing other cues will result in the reduction of necessary processing within the focused "pole." In these cases individuals may report being in a state of high, undivided presence (Biocca 2003, p. 7).

Tasks and environments can place high or low demands on spatial attention. For example, learning to ride a bicycle may be highly demanding of attention compared to sitting stationary with no current task may be less demanding. In cases where that physical environment is not demanding of spatial attention individual may experience low spatial presence and become disengaged. A mental imagery space (or thoughts) may dominate leading to an oscillation or division of Telepresence across two spatial models the physical and the imagery space. The phenomenal experience reported in self-report measures will be low spatial (physical) presence (Biocca 2003, pg 8).

Biocca maps how media channels might be places within the three pole model. He places virtual reality at the extreme end of virtual space and flight/fight responses at the extreme end of physical space. The result is that there is not a direct relationship between levels of Immersion and levels of Telepresence (Jones 2007). "Thus, regardless of how immersive or impoverished the medium is, the experience of Telepresence is determined by the quality of the physical, virtual, and/or mental spatial cues and the individual's awareness of them (Biocca 2003)" [as cited in Jones (2007), p. 41].

\subsection{Telepresence and Attention}

Researchers have long discussed a link between Telepresence and Attention. Witmer and Singer (1998) state that they view Telepresence as "a normal awareness phenomenon that requires directed attention and is based in the interaction between sensory stimulation, environmental factors that encourage involvement and enable immersion, and internal tendencies to become involve" (p. 225). They further attempt to conceptualize differences between Attention, Immersion, and Involvement. Witmer and Singer (1998) link attention to focus (Fontaine 1992). Focus refers to being aware of an entire environment and uses cues such as novelty to take in changes in the environment. In contrast, Witmer and Singer (1998) discuss focused attention as requiring most of one's attentional resources. This view is consistent with the Limited Capacity model (Lang 1990). Telepresence is seen as requiring both involvement and immersion (Witmer and Singer 1998): Involvement is defined as "a psychological state experienced as a consequence of focusing one's energy and attention on a coherent set of stimuli or meaningfully related activities and events" (Witmer and Singer 1998, p. 227). Whereas, Immersion is defined as "a 
psychological state characterized by perceiving oneself to be enveloped by, included in, and interacting with an environment that provides a continuous stream of stimuli and experience" (p. 227).

\subsubsection{Attention as secondary task reaction time}

According Basil (1994) secondary task reaction time (STRT) is capable of assessing "attention, arousal, and involvement" (Basil 1994, p. 93). STRT is an objective measure that is based on the limited capacity model of attention (Lang 1990). The basic assumptions are that people distribute their perceptual and cognitive resources to survey and interpret the world around them. These resources are limited and therefore, when resources are allocated to a specific channel, less are available to process other channels. STRT measures attention on this same premise that when participants are involved in an activity or task, then less attention is available for the other activities (i.e., secondary tasks). In a summary of STRT research. Lang et al. (1998) identify conditions that influence resource allocation: structural features (i.e. screen size), changes in the difficulty of encoding the task (i.e. pace), and the concurrent demands for resources (i.e., primary and secondary task performance).

\subsubsection{Telepresence and STRT}

There have numerous calls to identify more objective measures of Telepresence. One approach is to use STRT to measure the amount of time it tasks to distract participants from the media experience. Periodically, there has been interest in this approach. Darken et al. (1999) reported that "attention is a likely candidate measurement that correlated to a well-accepted self-assessment measurement of presence" (pg. 346).

There have been several previous studies that have employed STRT using a visual stimulus. For example Klimmt et al. (2005a, b) used a visual distraction cue in an experiment testing three levels of immersive media: hypertext, film, and VR. The authors' reported weak but negative correlations between attentional measures and the STRT responses. More recently, Nordahl and Korsgaard (2010) used a technique related to STRT called adjustable distraction to measure Telepresence. The results suggest that distraction cues may be useful for studying Telepresence because participants appear to be able to adjust to have multiple visual cues without disrupting their telepresence experience.

Bailey et al. (2011) employed aural audio distraction cues in an experiment testing Telepresence over the duration of series of film clips. The authors' did not measure the length of time it took participants to respond but instead coded either a failure to respond or that the cue elicited a response regardless of time. When participants reported experiencing a sensation of Telepresence there was " $\mathrm{min}$ imal failure" to respond to secondary task probes (pg. 13).

Based on the small but consistent trend in these studies that STRT can be used to test for Telepresence experiences, we posit the following;

H1 Attention (STRT) will be correlated to self-reported Telepresence sub-dimensions.

\section{Methods}

The data in this study was part of a larger study examining impact of content and form on telepresence experiences. The prior study utilized a $(2 \times 2)$ screen size by content/ pacing between-participant design with a covariate. Participants viewed either a large or small screen presentations of fast-paced action adventure content, or slowerpaced, conversation-driven content. In the current study, all conditions were combined. Ten-minute film clips were viewed by 120 participants with full Institutional Review Board approval. There were 12 audio distraction cues presented randomly throughout the 10 minutes.

\subsection{Participants}

Undergraduate students were recruited from several social science courses to participate. The 120 subjects were given credit from their instructors for participation. The majority of the group was female $(n=80)$.

\subsection{Stimulus}

The video material was taken from the film Ronin. The movie released in 1998 was directed by John Frankenheimer and starred Robert De Niro. After viewing several movies that included both character conversational interaction and scenes with high action sequences without (or with minimal) character conversation, this movie was selected. The content (pace) of each clip was different. The action/chase clip was fast paced with frequent cuts and lots of movement on the screen. The conversation clip is presented in a much slower pace. The scenes feature longer shot lengths and slower transitions.

\subsection{Independent variables}

\subsubsection{STRT}

Using Inquisit, we presented 12 audio distraction cues across the 10 minutes video clip. The participants were 
instructed they would hear an audio distraction cue and when they hear it they should press either the spacebar or the mouse key. The participants were provided with a practice video clip featuring a comedian telling jokes and during this video had three practice distraction cues. The distraction cue was a trumpet playing music.

We calculated reaction time latency means for each of the 120 participants across the 12 distraction cues. We excluded 11 participants who never hit the space bar or mouse during the 10 minutes video clip. This latency mean is what is used in the subsequent analyses.

\subsection{Dependent variables}

\subsubsection{Telepresence}

The amount of telepresence experienced by the participants was measured using items from the multidimensional telepresence scale (Bracken 2005, 2006; Lombard et al. 2009). The Telepresence items from the questionnaire were factor analyzed and resulted in four factors. These factors are similar to the sub-dimensions of Telepresence identified by Lombard and Ditton (1997). The factors are immersion, social realism, spatial presence, and transportation. Here transportation is consistent with a sense of "being there" previously identified by Kim and Biocca (1997).

\subsubsection{Immersion}

Immersion was measured by asking participants to respond to five statements using a scale from not at all (1) to very much (7). The items include "How involving was the video?" "How engaging was the story?" "How exciting was the experience?" "To what extent did you feel mentally immersed in the video?" $(\alpha=.86)$.

\subsubsection{Social Realism}

Social Realism was measured by asking participants to respond to three statements using a scale from not at all (1) to very much (7). The items include, "The way the events occurred were a lot like the ways those events occur in the real world." "The events in the video could occur in the real world." "It is likely the events in the video would occur in the real world. $(\alpha=.87)$.

\subsubsection{Spatial Realism}

Spatial Realism was measured by asking participants to respond to three statements using a scale from not at all (1) to very much (7). The items include, "How often did it feel as if you could reach out and touch objects or individuals in the experience?" "How often did an object heading toward you make you feel as if you should move?" "How much did it feel like the objects and events you viewed were all around you?" ( $\alpha=.78)$.

\subsubsection{Transportation}

Transportation was measured by asking participants to respond to three statements using a scale from not at all (1) to very much (7). The items include "Did you ever feel that your body was in the room, but your mind was in the media experience?" "To what extent did you experience a sense of Being There inside the media experience?" "How much did your feel as though you were inside the media experience observing the events?" $(\alpha=.88)$.

\subsubsection{Enjoyment}

Enjoyment was measured by asking participants to respond to two statements using a scale from not at all (1) to very much (7). The items include "Overall how satisfactory was the media experience?" "How enjoyable was the story?" $(\alpha=.58)$.

\section{Results}

Hypothesis 1 predicted that Attention (STRT) will be related to sub-dimensions of Telepresence. There was a significant relationship between Attention (as a covariate) and Telepresence-Immersion ( $F=10.33, p<.001$; see Table 1 ).

As expected, Attention (STRT) was correlated with the pencil-and paper measure of Immersion $(r=.30, p<.01)$. While significant, the linear relationship between Attention (STRT) and the paper and pencil Immersion index accounted for less that 10 percent of the relationship $\left(r^{2}=.09\right)$.

To further examine this relationship a correlation matrix was run to examine the relationship reaction time and the individual items of the Immersion scale. The results show that Attention (STRT) is correlated to the items that

Table 1 Multiple analysis of variance table for attention, image size and pace

\begin{tabular}{lcccc}
\hline & Sum of squares & $d f$ & Mean square & \multicolumn{1}{l}{$F$} \\
\hline Immersion & 235.80 & 1.00 & 235.80 & $10.33^{* * *}$ \\
Social Reality & .45 & 1.00 & .45 & .03 \\
Spatial & .64 & 1.00 & .64 & .05 \\
Transportation & 17.14 & 1.00 & 17.14 & .98 \\
Enjoy & 8.84 & 1.00 & 8.84 & 1.02 \\
\hline
\end{tabular}

$* * * p<.001$ 
Table 2 Correlations between STRT, immersion items and overall enjoyment

$p<.05 ; * * p<.01 ;$ **** $p<.001$

measure immersion. Specifically, there are negative correlations to items that inquire about involvement, engagement, excitement, and mental immersion. So, when a participant was involved, engaged, found the content exciting or was mentally immersed their reaction time was slower (see Table 2).

\section{Discussion}

The current study revisited the use of STRT to measure Telepresence. The results indicate that there is value in using this measurement technique for some of the subdimensions of Telepresence. Specifically, the analyses point to the relationship between Attention and Immersion. Using STRT as an indicator of Attention, we attempted to disentangled the conventional Immersion index to examine the components relationship with a conventional non-self report measure of Attention.

The correlations of the individual Immersion items and reaction time should be very intriguing both to Telepresence and Attention researchers. The correlations are significant and suggest that the variables share an element in regards to what they measure. However, if the variables were measuring the same thing, one might expect the correlations to be higher. We see that generally the Immersion items go better with one another than they do with the attention measure. These zero order relationships suggest that there may be distinctions between Immersion, Attention, and Enjoyment, similar to those suggested by Witmer and Singer (1998). It also suggests that some of the Telepresence measurements might be masking other relationships that have been occurring. An examination of the conventionally combined Telepresence indices may bear fruit in our attempt to better define the concepts of telepresence.

This study suggests that the various dimensions (spatial, social realism and enjoyment) of Telepresence commonly used may be artifacts of the particular "story being told" in the mediated experience. But just as good characters and good story can create attention and immerse one in a story, no one element necessarily alone (or in simple combination) predicts the level of Telepresence of a mediated experience. If Attention and Immersion are related but somewhat different, then perhaps there are two dimensions: one has a certain intentionality (Attention/Cognitive) and one is created via media characteristics (Immersion/Emotive). If one needs to understand elements of a message then an immersive message might assist Attention and understanding, but a message with immersive characteristics may not be necessary for the attention of understanding. If one is to be tested on a piece of dry textual writing, directed cognitive energy may surfice. On the other hand if the message is immersive, cognitive intentionality may not be necessary to establish a sense of Telepresence in the experience. For example, action films can be immersive with minimal story or character development.

It appears that Immersion is capturing elements that pull media users into the media environment. Of the variables measured in this study it is the closest to attention. We feel that these results suggest that there may be emotional reactions that work independently with the cognitive processing. There may be presentational elements that demand a reaction and that immersion appears to capture some of this reaction. People have instinctual responses to media environments and content.

Additionally, this view is at least in part consistent with Reeves' (1991) ideas about being there. Steuer (1992) quotes Reeves as saying being there is "a combination of automatic perceptual processes, mindful direction of attention, and conscious processes such as narratization, all contribute toward our perceiving mediated experiences as if they were real" (Reeves 1991, p. 6).

These results point out the need for further investigation of attentional processing in communication and telepresence research. Finally, we think this may linkage may well be elaborate the traditional Attentional Model in conventional media use but also add a way for the conventional models to better assimilate the new media experiences that seem to emerge almost daily.

\section{Conclusion}

The use of STRT may be beneficial in some types of Telepresence research. We encourage presence researchers to continue to employ objective measures in their research. Additionally, the link between Attention and Telepresence needs further investigation. 


\section{References}

Alcañiz M, Rey B, Tembl J, Parkhutik V (2009) A neuroscience approach to virtual reality experience using transcranial Doppler monitoring. Presence Teleoper Virtual Environ 18(2):97-111

Bailey RL, Rubenking B, Lang A (2011) The influence of trait motivational reactivity on the formation of motivated cognitive states: flow, presence, and transportation. In: Simmons RR (ed) Psychophysiology, 48, supplement 1. Society for Psychophysiological Research, S103

Basil MD (1994) Secondary reaction-time measures. In: Lang A (ed) Measuring psychological response to media. Lawrence Erlbaum Associates, Mahwah, pp 85-98

Biocca F (2003) Can we resolve the book, the physical reality, and the dream state problems? From the two-pole to a three-pole model of shifts in presence. Presented at the EU Future and Emerging Technologies Presence Initiative Meeting, Venice, 5-7 May 2003. Available at http://www.mindlab.org/images/d/DOC705. pdf

Bracken CC (2005) Presence and image quality: the case of high definition television. Media Psychol 7(2):191-205

Bracken CC (2006) Perceived source credibility of local television news: the impact of image quality and presence. J Broadcast Electron Media 50(4):723-741

Bracken CC, Pettey G, Guha T, Rubenking BE (2010) Sounding out small screens and telepresence: the impact of audio, screen size, and pace. J Media Psychol Theor Methods Appl 22(3):125-137

Darken RP, Bernatovich D, Lawson J, Peterson B (1999) Quantitative measures of Presence in virtual environments: the roles of attention and spatial comprehension. Cyberpsychol Behav $2: 337-347$

Fontaine G (1992) The experience of a sense of presence in intercultural and international encounters. Presence Teleoperators Virtual Environ 1(4):482-490

IJsselsteijn WA, de Ridder H, Freeman J, Avons SE (2000) Presence: concept, determinants and measurement. In: proceedings of the SPIE 3959:520-529. Available Online: http://www.presenceresearch.org/papers/SPIE_HVEI_2000.pdf. Retrieved April 18, 2011

Jones MT (2007) Presence as external versus internal experience: how form, user, style, and content factors produce presence from the inside. In: Proceedings of the Annual International Workshop on Presence. Available at http:/www.temple.edu/ispr/prev conferences/proceedings/2007/Jones.pdf

Kim T, Biocca F (1997) Telepresence via television: two dimensions of telepresence may have different connections to memory and persuasion. J Comput Mediat Commun 2(3)

Klimmt C, Hartmann T, Gysbers A, Voderer P (2005) The value of reaction-time measures in presence research: Empirical findings and future perspectives. In: proceedings of the annual presence conference pp 293-298

Klimmt C, Gysbers A, Hartmann T, Nosper A, Behr K, Vorderer P (2005b) Do secondary task reaction times measure (precursors of) spatial presence? Paper presented at the International Communication Association, New York

Lang A (1990) Involuntary attention and physiological arousal Commun Res 17(3):275

Lang A, Basil MD, Roloff MF (1998) Attention, resource allocation, and communication research: what do secondary task reaction times measure anyway? Commun Yearb 21:443-473

Lombard M, Ditton TB (1997) At the heart of it all: the concept of presence. J Comput Mediat Commun 3(2)

Lombard M, Ditton TB, Weinstein L (2009) Measuring (tele)presence: the temple presence inventory. Presented at the Twelfth International Workshop on Presence, Los Angeles, California, USA

Nordahl R, Korsgaard D (2010) Distraction as a measure of presence: using visual and tactile adjustable distraction as a measure to determine immersive presence of content in mediated environments. Virtual Real 14:27-42

Pettey P, Bracken C, Rubenking B, Buncher M, Gress E (2010) Telepresence, soundscapes and technological expectation: putting the observer into the equation. Virtual Real 14(1):15-25. doi: 10. 1007/s 10055-009-0148-8

Pinker S (2007) The language instinct: how the mind creates language. Harper Perennial Modern Classics, New York

Reeves BR (1991) Being there: television as symbolic versus natural experience. Unpublished manuscript. Stanford University, Institute for Communication Research, Stanford

Steuer J (1992) Defining virtual reality: dimensions determining telepresence. J Commun 4(24):73-93

Witmer B, Singer MJ (1998) Measuring presence in virtual environments: a presence questionnaire. Presence 7(3):225-240 